\title{
Investigation of the Effect of Microstructure and Grain Boundaries in Nanostructured CMR Thin Films Using Scanning Tunneling Microscopy (STM) and Local Conductance Map (LCMAP)
}

\author{
Sohini Kar, Jayanta Sarkar, and A. K. Raychaudhuri
}

\begin{abstract}
We have investigated the spatially resolved local electronic properties of a nanostructured film of a colossal magnetoresistive (CMR) material by local conductance mapping (LCMAP) using a variable temperature scanning tunneling microscope (STM) operating in a magnetic field. The nanostructured thin films (thickness $\approx 500 \mathrm{~nm}$ ) of the CMR material $\mathrm{La}_{0.67} \mathrm{Sr}_{0.33} \mathrm{MnO}_{3}$ (LSMO) on silicon substrates were prepared using chemical solution deposition (CSD) process. These films have a large density of natural incoherent grain boundaries (GBs) which leads to significantly different behavior compared to oriented and epitaxial films of the same composition. Due to the presence of the GBs, these films show substantial low field magnetoresistance (LFMR) followed by a slower and almost linear decrease at higher fields and this is found to be strictly dependent on particle size. Most of the mechanisms proposed to explain the LFMR in the GB are based on tunneling through the GB. The purpose of this study is to use different STM based techniques to image these inhomogeneities and quantify them to the extent possible. In particular, we study the effect of grain size and the grain boundaries and their role in the electrical transport in nanostructured films of CMR materials.
\end{abstract}

Index Terms-Colossal magnetoresistance, grain boundaries, nanostructured films, scanning tunneling microscopy.

\section{INTRODUCTION}

$\mathbf{H}$ OLE-DOPED manganites are widely investigated because they exhibit colossal magnetoresistance (CMR) [1]-[4]. CMR in these materials refers to the suppression of the resistivity upon application of a magnetic field. This makes them attractive candidates for application in magnetic field sensing and magnetic recording devices. They have a general chemical formula $\mathrm{Re}_{1-x} \mathrm{~A}_{x} \mathrm{MnO}_{3}$ (where Re is a rare earth element and $\mathrm{A}$ is a divalent element like $\mathrm{Sr}, \mathrm{Ca}, \mathrm{Ba}, \mathrm{Pb}$, etc.). They show a transition from a paramagnetic insulating state (PMI) to a ferromagnetic metallic state (FMM) at a characteristic temperature $\left(T_{c}\right)$. These materials also show a transition from

Manuscript received April 5, 2006; revised July 5, 2006. This work was supported in part by the Center for Scientific and Industrial Research (CSIR), India, and in part by the DST Unit for Nanoscience, S. N. Bose National Centre for Basic Sciences. The review of this paper was arranged by Associate Editor S. Khizroev.

S. Kar and J. Sarkar are with the Department of Physics, Indian Institute of Science, Bangalore 560012, India.

A. K. Raychaudhuri is with the DST Unit for Nanoscience, S. N. Bose National Centre for Basic Sciences, Kolkata 700098, India

Color versions of Figs. 1-4 are available online at http://ieeexplore.ieee.org.

Digital Object Identifier 10.1109/TNANO.2006.883483 a high-temperature semiconducting phase to a low-temperature metallic phase at a temperature close to $T_{c}$. Generally, a peak in the resistivity occurs at the transition. We refer to the temperature at which a peak in the resistivity occurs as $T_{p}$ (where $\left.T_{p} \approx T_{c}\right)$.

Of particular interest in this field is the growth of high-quality manganite thin films having appreciable magnetoresistance and $T_{c}$ higher than room temperature $(300 \mathrm{~K})$ for potential practical applications. It has been established through studies on artificial grain boundary devices and polycrystalline materials of different grain sizes that grain boundaries play an important role in the electrical transport properties [5], [6]. Nanostructured materials are interesting sensor materials because they exhibit substantial magnetoresistance (MR) over an appreciable temperature range below $T_{c}$.

There have been a significant number of investigations on oriented and epitaxial films of manganites in the past [7]-[9]. However, very little attention has been given to nanostructured films of the same composition. These thin films have a large density of incoherent natural GBs which leads to different behavior as compared to oriented and epitaxial films. We have carried out transport measurements in nanostructured films having different average particle sizes and observe that these films exhibit significant LFMR over an extensive temperature range. This is in contrast to single crystals or epitaxial films of the same composition, where the MR peaks near $T_{c}$ but is very small at lower temperatures. The presence of the GBs also makes the transport in these films rather inhomogeneous. We investigate these inhomogeneities using different STM-based techniques. In particular we use a technique called local conductance mapping in which we make a simultaneous recording of the topography as well as a map of the differential tunneling conductance across the entire topographic scan range. This method allows us to identify different conductance regions in the film and to study the local electronic properties.

\section{EXPERIMENT}

The nanostructured thin films of $\mathrm{La}_{0.67} \mathrm{Sr}_{0.33} \mathrm{MnO}_{3}$ (LSMO) of nominal thickness $\approx 500 \mathrm{~nm}$ were used in the present investigation. They were prepared by chemical solution deposition (CSD) route on silicon substrates [10], [11] using precursors dissolved in acetic acid and water. The details of the sample preparation are given elsewhere [12]. The films thus formed were studied using X-ray diffraction (XRD) to confirm phase 
formation and purity, and atomic force microscopy (AFM) to characterize the surface morphology [13]. By varying the parameters such as annealing time and temperature, nanostructured films having different average grain sizes can be fabricated. For this investigation we studied two LSMO nanostructured films having average grain size 70 and $150 \mathrm{~nm}$. The grain sizes were determined by AFM analysis. The transport properties of these films were measured using standard four-probe technique in the temperature range $1.4 \mathrm{~K}-300 \mathrm{~K}$ and in magnetic fields up to $12 \mathrm{~T}$.

The spatially resolved measurements on the nanostructured LSMO films were carried out using a home made high vacuum low-temperature scanning tunneling microscope (LTSTM) operating in a magnetic field. The scans were taken at different temperatures above and below $T_{p}$ of the LSMO thin films and also in different magnetic fields. The present paper is more focused on the local differential conductance mapping (LCMAP), in which we made a simultaneous recording of the topographic image as well as a map of the differential conductance $g(V)=$ $d I / d V$ (taken at a given bias) across the entire topographic scan range. In this case $g(V)$ is also a function of the spatial coordinates. The LCMAP is thus a two-dimensional (2-D) map of the tunneling conductance $g(V)$ across the surface for a fixed bias and at a fixed average tunnel current. The details of the experimental setup are given elsewhere [14]. Briefly, the dc bias to the sample is modulated by a small ac signal $\left(\delta V_{\mathrm{ac}} \ll\right.$ $\left.V_{\mathrm{dc}}\right)$. The resulting tunnel current now has an additional ac component $\left(\delta I_{\mathrm{ac}}\right)$ at the ac excitation frequency which was measured using a lock-in amplifier giving the $d I / d V$ at $V_{\mathrm{dc}}$ directly. The dc component of the tunnel current was used to keep the feedback loop active and this generated the topographic image while $d I / d V$ from the lock-in was used to generate a simultaneous conductance map of the scan area. The ac modulation frequency was chosen such that it was much higher than the feedback cutoff frequency so that the change in modulation in the tunnel current was not compensated for by the feedback loop. At such biases, $g(V)=d I / d V$ is the tunneling conductance and $g(V) \propto G(E)$, the surface density of states, where $E=e V_{\mathrm{dc}}$. The more metallic regions on the film have higher surface density of states and subsequently higher conductance, which show up as brighter areas on the LCMAP. The contrast in the LCMAP is thus a measure of the variation in $G(E)$ across the particular scan area and we are therefore able to identify and separate the high conductance regions from the low conductance ones.

\section{RESULTS AND DISCUSSIONS}

The resistances versus temperature graphs of the two films are shown in Fig. 1(a) and (b). The transition temperatures, $T_{p}$, as obtained from the transport measurements done on the film were about $200 \mathrm{~K}$ for the LSMO thin film having average grain size of $70 \mathrm{~nm}$ [see Fig. 1(a)] and about $250 \mathrm{~K}$ for the LSMO thin films having grain size of about $150 \mathrm{~nm}$ [see Fig. 1(b)]. Both films show a very sharp upturn in resistivity as we lower the temperature below $30 \mathrm{~K}$, where the resistivity is seen to increase sharply. This can be attributed to the presence of the GBs. The transport across the GB is believed to arise from multistep tunneling process [7]. Fig. 1 also shows the change in resistance

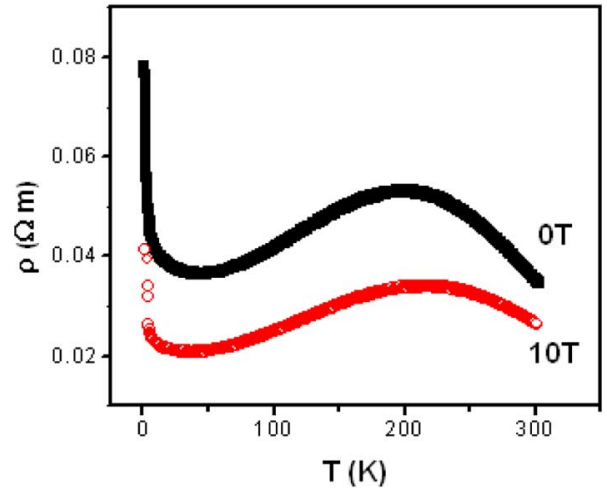

(a)

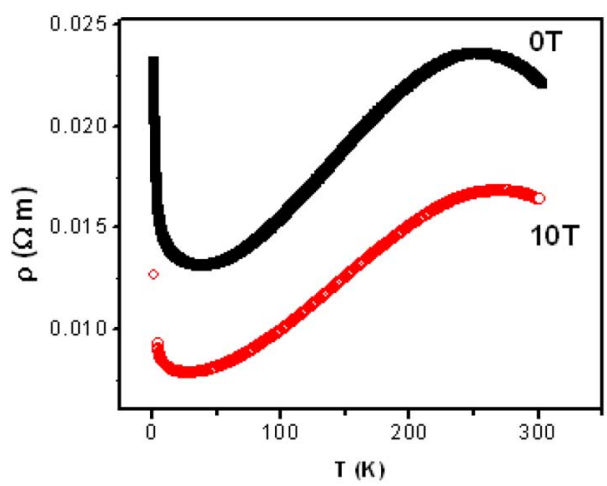

(b)

Fig. 1. Resistivity as a function of temperature in zero field and $10 \mathrm{~T}$ of the LSMO nanostructured thin films having average grain size of: (a) $70 \mathrm{~nm}$ and (b) $150 \mathrm{~nm}$.

as a function of temperature in a magnetic field of $10 \mathrm{~T}$. It can be seen that the resistivity decreases in both the films as the magnetic field is increased. The MR, as can be seen from the data is substantial over the whole temperature range below $T_{p}$ and it is appreciable even above $T_{p}$.

In Fig. 2 we show the topographic images and the corresponding LCMAPs of the two films as obtained from the STM slightly above room temperature $(T=315 \mathrm{~K})$.

One of the most interesting transport properties observed in nanostructured manganite thin films is the occurrence of low field magnetoresistance (LFMR) which increases as the temperature decreases. We have measured the LFMR from $1.4 \mathrm{~K}$ to 100 K. In Fig. 3 we show a typical data set for LFMR measured at fields up to $1 \mathrm{~T}$ in the case of the thin film having average grain size of $70 \mathrm{~nm}$. We observe that the LFMR is about $25 \%$ at $1 \mathrm{~T}$ and $1.4 \mathrm{~K}$ and this decreases to about $12 \%$ at $100 \mathrm{~K}$ for the $70 \mathrm{~nm}$ grain size film. These values of LFMR are somewhat higher in the case of the $150 \mathrm{~nm}$ grain size film.

Simultaneous topographic and conductance maps were taken using the STM at different temperatures above and below $T_{p}$ of the LSMO thin films. Fig. 4 shows typical sets of LCMAPs obtained at three different temperatures chosen to be above, below and around $T_{p}$, for both the films. Fig. 4(a) shows LCMAPs taken on $200 \mathrm{~nm} \times 200 \mathrm{~nm}$ scan areas on the LSMO thin film having average grain size $70 \mathrm{~nm}$ while Fig. 4(b) shows LCMAPs taken on $500 \mathrm{~nm} \times 500 \mathrm{~nm}$ scan areas on the LSMO thin films having average grain size $150 \mathrm{~nm}$. The brighter areas on the images correspond to regions of higher conductance while the darker areas correspond to regions of lower conductance. The 

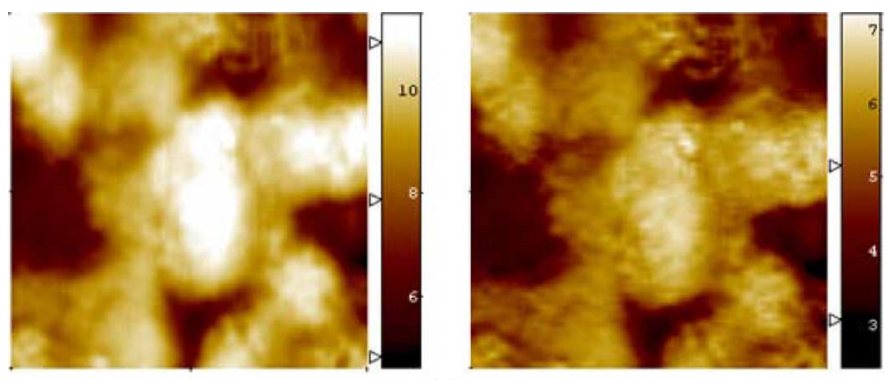

(a)
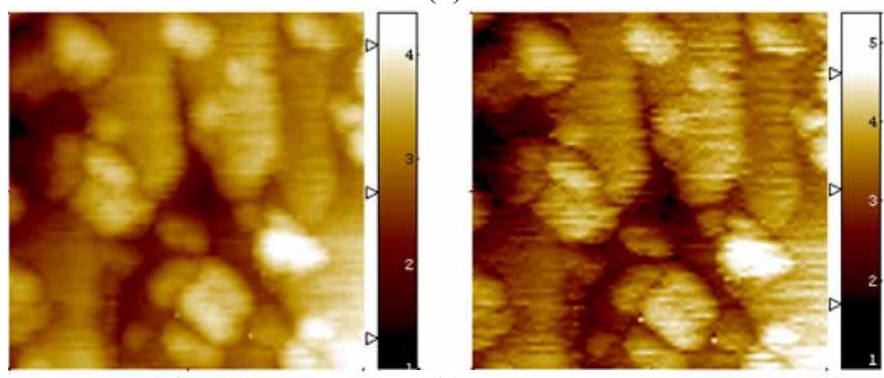

(b)

Fig. 2. Topographic images (left panels) and corresponding LCMAPs (right panels) of the nanostructured films as obtained from the STM at $T=315 \mathrm{~K}$ : (a) $200 \mathrm{~nm} \times 200 \mathrm{~nm}$ image of thin film having average grain size of $70 \mathrm{~nm}$ and (b) $500 \mathrm{~nm} \times 500 \mathrm{~nm}$ image of thin film having average grain size of $150 \mathrm{~nm}$.

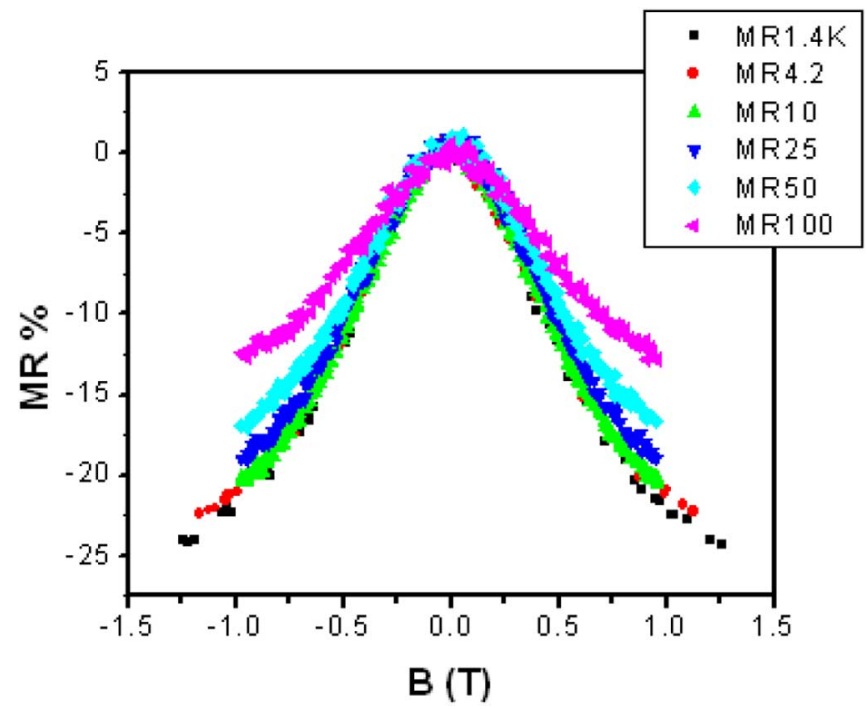

Fig. 3. Low field magnetoresistance at different temperatures in LSMO nanostructured thin film having average grain size $70 \mathrm{~nm}$.

grains and GBs are separately identified by taking several line scans across the topographic image, which bears a strong correlation to the corresponding LCMAP [15].

When we compare the LCMAPs with the corresponding topographic scans, we observe that there are sharp drops of conductance at the grain boundaries whereas the conductance is higher and relatively constant within the grain. The GBs therefore form a low conductance region leading to inhomogeneity in the transport through the film. LCMAP gives the local tunneling conductance which is proportional to the density of states (DOS) near the Fermi level. The low (high) conductance regions are expected to have low (high) DOS leading to lower (higher) tunneling conductance. An interesting way to see the inhomogeneous nature of the transport in these nanostructured films is to

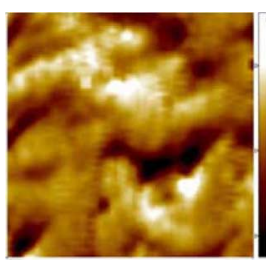

$\mathrm{T}=86 \mathrm{~K}$

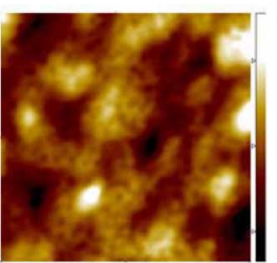

$\mathrm{T}=78 \mathrm{~K}$

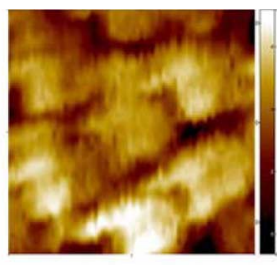

$\mathrm{T}=210 \mathrm{~K}$

(a)

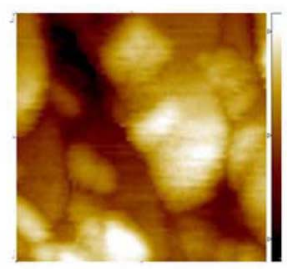

$\mathrm{T}=251 \mathrm{~K}$

(b)

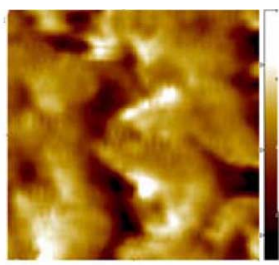

$\mathrm{T}=297 \mathrm{~K}$

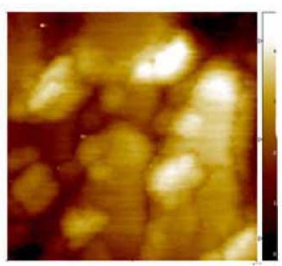

$\mathrm{T}=295 \mathrm{~K}$
Fig. 4. Typical sets of LCMAPs taken at different temperatures above, below and around the transition temperature $T_{p}$, for LSMO thin films having average grain size of: (a) $70 \mathrm{~nm}$ and (b) $150 \mathrm{~nm}$.

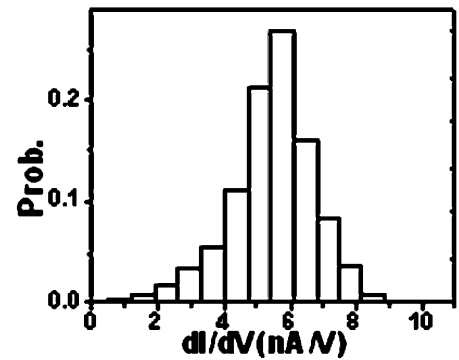

(a)

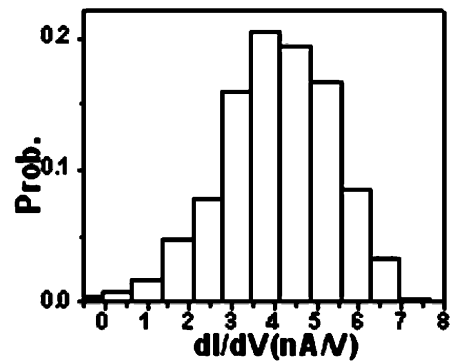

(b)

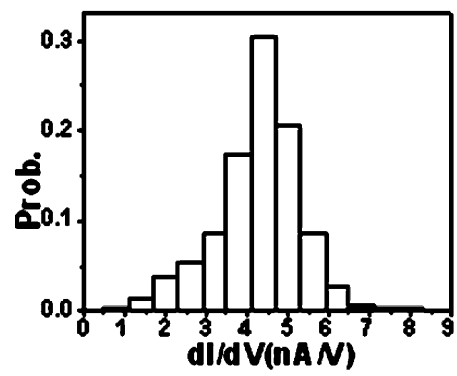

(c)

Fig. 5. Typical sets of conductivity histograms taken at different temperatures: (a) $T=86 \mathrm{~K}$, (b) $T=210 \mathrm{~K}$, and (c) $T=297 \mathrm{~K}$, for LSMO thin film having average grain size $70 \mathrm{~nm}$.

follow the conductance at different regions of the film as a function of temperature. For this we constructed a local tunneling conductivity histogram from the LCMAP data. The typical data set is shown in Fig. 5 for the nanostructured film having average 


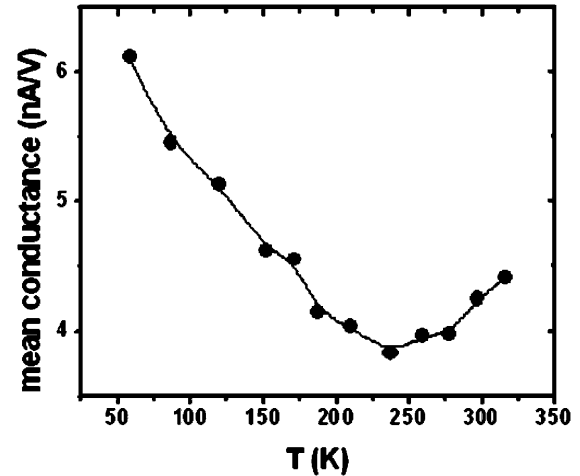

(a)

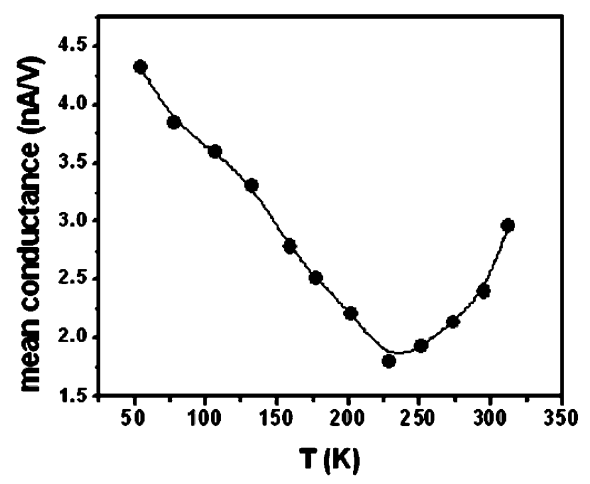

(b)

Fig. 6. Variation of the mean conductance as a function of temperature for LSMO thin films having average grain size of: (a) $70 \mathrm{~nm}$ and (b) $150 \mathrm{~nm}$.

grain size $70 \mathrm{~nm}$. One can clearly observe that the local tunneling conductance has a wide distribution and both the mean as well as the width of the distribution shifts as the temperature is lowered through $T_{p}$. This establishes that the GBs and the grains not only have different conductances but the temperature dependences are also different.

In order to quantify the degree of fluctuations in the local conductance we calculated the mean local conductance and standard deviations (SD) of the fluctuations $\left\langle\delta g^{2}\right\rangle^{0.5}$ variation of $\langle g\rangle$ as a function of temperature for the two from the line scans across the LCMAPs. Fig. 6 shows variation of $\langle g\rangle$ as a function of temperature for both the films. Both the films show a gradual decrease as we reduce the temperature followed by an upturn around the transition temperature. The mean conductance $\langle g\rangle$ is weighted preferentially by the conductances from regions that have high $\langle g\rangle$ values. These are the grains. The change in $\langle g\rangle$ on application of field will thus be more affected by the contribution of the grains. In grains the MR peaks near $T_{p}$. The observed dipping of $\langle g\rangle$ near $T_{p}$ thus confirms this.

The standard deviation in conductance taken from several line scans for both LSMO thin films is shown in Fig. 7. The curves have rather sharp changes near the $T_{p}$ and then they increase slowly with decreasing temperature. This means that as the temperature is lowered below $T_{p}$, the contrast between the grains and the GBs is stronger and the conduction tends to become more inhomogeneous with the GBs contributing more prominently to the electrical transport, and this manifests itself in the observation of LFMR in these films which becomes more prominent at lower temperatures. We also note a very important observation. The

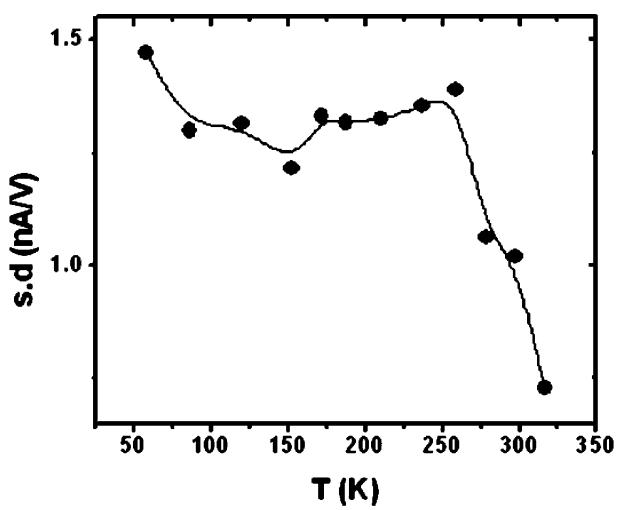

(a)

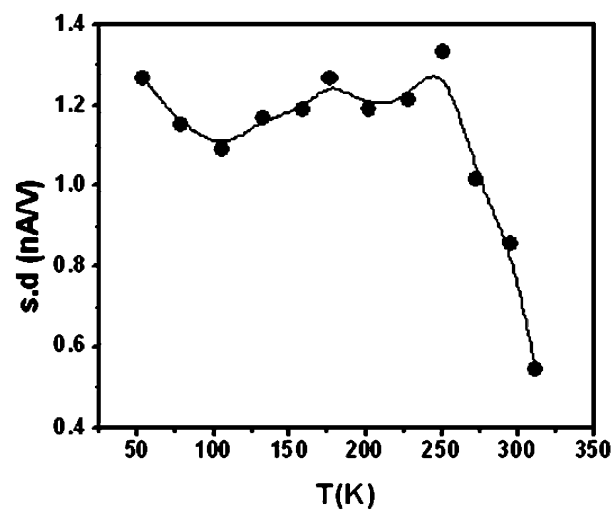

(b)

Fig. 7. Variation of standard deviation in mean conductance as a function of temperature for LSMO thin films having grain size of: (a) $70 \mathrm{~nm}$ and (b) $150 \mathrm{~nm}$.

standard deviation shows a clear upturn at temperatures below $100 \mathrm{~K}$. In this region the resistance also shows an upturn. This presumably happens because at lower temperatures the GB region makes dominant contribution to transport. Since the transport across the GB region is a tunneling process it leads to an enhancement of GB resistance at lower temperatures. This enhances the film resistance and also makes the conductance distribution more skewed, thus increasing the standard deviation.

\section{CONCLUSION}

We have studied the transport properties of nanostructured thin films of the CMR material $\mathrm{La}_{0.67} \mathrm{Sr}_{0.33} \mathrm{MnO}_{3}$ having different average grain sizes and probed the local electronic properties using STM-based techniques. We were able to spatially resolve the local differential surface conductance using LCMAPs. The variation with temperature of the mean tunneling conductance, which is proportional to the surface density of states, shows that transport within the grains translates to the bulk resistivity seen in these samples. The contrast in the LCMAPs, quantified by the standard deviation in the conductance shows the increased contribution from the grain boundary regions as we lower the temperature and hence its contribution to the inhomogeneities in transport properties and the LFMR observed in these nanostructured films, which is otherwise absent in oriented and epitaxial films of the same contribution. We also observe the change in transition temperature with grain size which suggests that the transport properties of these films can be tuned to a large extent by proper control of the grain size. 


\section{REFERENCES}

[1] R. v. Helmolt, J. Wecker, B. Holzapfel, L. Schultz, and K. Samwer, "Giant negative magnetoresistance in perovskitelike $\mathrm{La}_{2 / 3} \mathrm{Ba}_{1 / 3} \mathrm{MnO}_{x}$ ferromagnetic films," Phys. Rev. Lett., vol. 71, pp. 2331-2333, 1993 .

[2] Y. Tokura, Ed., Colossal Magnetoresistive Oxides. Singapore: Gordon and Breach Science, 2000.

[3] K. Chahara, T. Ohno, K. M. Kasai, and Y. Kozono, "Magnetoresistance in magnetic manganese oxide with intrinsic antiferromagnetic spin structure," Appl. Phys. Lett., vol. 63, pp. 1990-1992, 1993.

[4] C. Rao and B. Raveau, Eds., Colossal Magnetoresistance, Charge Ordering and Related Properties of Manganese Oxides. Singapore: World Scientific, 1998.

[5] N. D. Mathur, G. Bumell, S. P. Isaac, T. J. Jackson, B.-S. Teo, J. S. MacManus-Driscoll, L. F. Cohen, J. E. Evetts, and M. G. Blamire, "Large low-field magnetoresistance in $\mathrm{La}_{0.7} \mathrm{Ca}_{0.3} \mathrm{MnO}_{3}$ induced by artificial grain boundaries," Nature (Lond.), vol. 387, pp. 266-268, 1997.

[6] R. Mahesh, R. Mahendiran, A. K. Raychaudhuri, and C. N. R. Rao, "Effect of particle size on the giant magnetoresistance $\mathrm{La}_{0.7} \mathrm{Ca}_{0.3} \mathrm{MnO}_{3}$," Appl. Phys. Lett., vol. 68, pp. 2291-2293, 1996.

[7] M. Paranjape, J. Mitra, A. K. Raychaudhuri, N. K. Todd, N. D. Mathur, and M. G. Blamire, "Nonlinear electrical transport through artificial grain boundary junctions in $\mathrm{La}_{0.7} \mathrm{Ca}_{0.3} \mathrm{MnO}_{3}$ epitaxial thin films," Phys. Rev B, vol. 68, pp. 144 409-144 416, 2003.

[8] R. A. Rao, D. Lavric, T. K. Nath, C. B. Eorn, L. Wu, and F. Tsui, "Three-dimensional strain states and crystallographic domain structures of epitaxial colossal magnetoresistive $\mathrm{La}_{0.8} \mathrm{Ca}_{0.2} \mathrm{MnO}_{3}$ thin films," Appl. Phys. Lett., vol. 73, pp. 3294-3296, 1998.

[9] M. Paranjape, A. K. Raychaudhuri, N. K. Todd, N. D. Mathur, and M. G. Blamire, "Effect of strain on the electrical conduction of epitaxial films of $\mathrm{La}_{0.7} \mathrm{Ca}_{0.3} \mathrm{MnO}_{3}$," Phys. Rev B., vol. 67, pp. 214415-214 420, 2003.

[10] T. Kiyotaka, S. Okamura, and T. Shiosaki, "Fabrication of perovskite manganite ( $\mathrm{La}, \mathrm{Sr}) \mathrm{MNO}_{3}$ thin films by chemical solution deposition and their magnetoresistance properties at room temperature," Jpn. J. Appl. Phys, vol. 40, pp. 6821-6824, 2001.

[11] A. D. Polli, F. F. Lange, M. Ahlskog, R. Menon, and A. K. Cheetam, "Processing magnetoresistive thin films via chemical solution deposition," J. Mater. Res., vol. 14, pp. 1337-1342, 1999.

[12] B. Ghosh, L. K. Brar, and A. K. Raychaudhuri, "Growth of oriented films of $\mathrm{La}_{0.67} \mathrm{Ca}_{0.33} \mathrm{MnO}_{3}$ and $\mathrm{La}_{0.67} \mathrm{Sr}_{0.33} \mathrm{MnO}_{3}$ on $\mathrm{SrTiO}_{3}$ using chemical solution deposition," J. Phys. D, Appl. Phys., vol. 37, pp. 1548-1533, 2004.

[13] B. Ghosh, S. Kar, L. K. Brar, and A. K. Raychaudhuri, "Electronic transport in nanostructured films of $\mathrm{La}_{0.67} \mathrm{Sr}_{0.33} \mathrm{MnO}_{3}$," J. Appl. Phys. , to be published.

[14] J. Mitra, A spatially resolved spectroscopic investigation into the ferromagnetic metallic state in hole doped manganites. Bangalore, India, Indian Institute of Science, 2004, Ph.D.
[15] S. Kar, B. Ghosh, L. K. Brar, M. A. Paranjape, and A. K. Raychaudhuri, "Mapping of local electronic properties in nanostructured $\mathrm{cmr}$ thin films by scanning tunneling microscopy (STM) and local conductance map (LCMAP)," in Proc. Mater. Res. Soc. Symp., 2005, vol. 838E, pp. O10.13.1-O10.13.6.

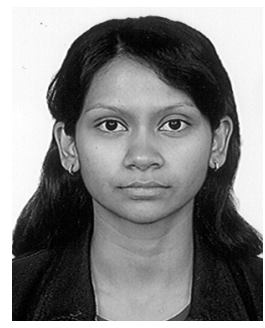

Sohini Kar received the M.S. degree with specialization in experimental condensed matter physics from the Indian Institute of Science, Bangalore, in 2004. She is currently working toward the Ph.D. degree in the Department of Physics, Indian Institute of Science.

Her current research interests include the study of spatially resolved electronic properties of nanostructured magnetic materials using scanning tunneling microscopy and the fabrication of nanoscale devices transport in metals and oxides. using e-beam lithography to study spin-polarized

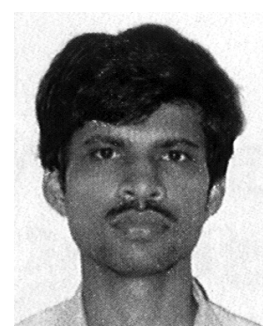

Jayanta Sarkar received the M.S. degree with specialization in experimental condensed matter physics from the Indian Institute of Science, Bangalore, in 2005. He is currently working toward the Ph.D. degree in the Department of Physics, Indian Institute of Science.

His research interests include growth and characterization of nanostructured manganite thin films and piezoresistive and bolometric applications of manganites.

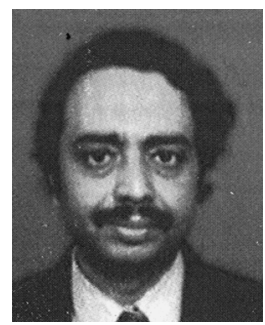

A. K. Raychaudhuri received the Ph.D. degree from Cornell University, Ithaca, NY, in 1980.

He was the Director of National Physical Laboratories, New Delhi, India, from 1997 to 2000 . He is currently the Director of S. N. Bose National Center for Basic Sciences, Kolkata, India. He is also a Professor at the Department of Physics, Indian Institute of Science, Bangalore. His main areas of research include scanning probe microscopy, low-temperature physics, physics of disordered materials, noise, and fluctuation and physics of novel nanomaterials.

Dr. Raychaudhuri is the recipient of several awards and fellowships. 